\title{
Response to Leptin and Nitric Oxide in Blood Pressure Regulation in Humans
}

\author{
Osama Alsmadi, ${ }^{1}$ Motasem Melhem, ${ }^{1}$ Prashantha Hebbar, ${ }^{2, *}$, Arshad Channanath, ${ }^{2}$ Gaurav Thareja, ${ }^{2}$ \\ Sumi E. John, ${ }^{2}$ Fadi Alkayal, ${ }^{1}$ Kazem Behbehani, ${ }^{3}$ and Thangavel Alphonse Thanaraj ${ }^{2}$
}

To the Editor: We appreciate the interest of Dr Tsuda in our article, "Leptin in association with common variants of MC3R mediates hypertension." We demonstrated, in a study population of Kuwaiti natives (of mean body mass index, $\mathrm{BMI}=32.20 \pm 8.41)$, significant association between MC3R variant (rs3827103 (Val81Ile)) and systolic blood pressure (SBP). Minor allele frequencies in study population of this and another MC3R variant (rs3746619 (Thr6Lys)), both of which are in high linkage disequilibrium with one another, are twice of those seen in European population. Presence of these 2 variants show a positive correlation with SBP; and leptin levels are seen positively correlated with SBP in individuals harboring the variants. It is further the case that MC3R expression is seen downregulated in such individuals. These results using human subjects are in agreement with reports from animal models that MC3R deactivation leads to hyperleptinemia. Based on the knowledge that leptin infusion can lead to an

*This author made significant intellectual and data contributions to this letter.

Correspondence: Thangavel Alphonse Thanaraj (alphonse.thangavel@dasmaninsitute.org).

'Dasman Genome Centre, Dasman Diabetes Institute, Kuwait; ${ }^{2}$ Integrative Informatics Department, Dasman Diabetes Institute, Kuwait; ${ }^{3}$ Director-General, Dasman Diabetes Institute, Kuwait.

Initially submitted July 27, 2014; date of first revision July 29, 2014; accepted for publication July 30, 2014.

doi:10.1093/ajh/hpu178

(c) American Journal of Hypertension, Ltd 2014. All rights reserved. For Permissions, please email: journals.permissions@oup.com increase in sympathetic nerve activity (SNA), which in turn leads to high blood pressure, we proposed a SNA-mediated mechanism to explain our observations. Dr Tsuda points out the mediation of nitric oxide (NO) in the regulation of hypertension in animal models-high leptin levels are known to lead to a higher concentration of NO, which in turn leads to relaxation of blood vessels (that can help to promote normal blood pressure). Dr Tsuda reiterates that release of NO from endothelial cells in response to increased leptin levels may contribute to the homeostasis of blood pressure. ${ }^{2}$

In our study, while we see that the positive correlation between BMI and leptin holds in the entire study population, positive correlation between leptin and SBP occurs only in individuals that harbor the MC3R variants (Figure 1). In a similar manner, we need to identify the physical and chemical stimuli that regulate the correlation between leptin and NO release in human. Thus, we agree with Dr Tsuda that further works are required with the study population (i) to identify more inclusively the mechanisms that modulate associations of leptin with SNA activation (causing increased blood pressure) and with increased NO release (causing counteracting vasodilatory effects) and (ii) to delineate their combined effects on regulation of human hypertension. As NO can also regulate SNA (by virtue of its ability to act as neurotransmitter in the brain) in the control of blood pressure, ${ }^{3}$ the suggested study becomes even more interesting.

Taking the discussions a step further: Obesity is a major cause for the metabolic syndrome, which leads to the development of hypertension and diabetes. These disorders share common pathways that include increases in sympathetic nervous system, renin angiotensin aldosterone system, oxidative stress, adipokines, insulin resistance, and peroxisome proliferator-activated receptors. The onset of one facilitates the onset of other; e.g., hypertension can augment 2-fold the association of higher BMI with younger age of type 2 diabetes onset. ${ }^{4}$ Identifying whether leptin can be a major link (among others) between human hypertension and diabetes is an open question. Such studies would benefit obese populations such as Kuwaitis who exhibit a high prevalence of diabetes (25.4\%), hypertension (28\%), and comorbidity $(11 \%) .^{5}$

\section{DISCLOSURE}

The authors declared no conflict of interest.

\section{REFERENCES}

1. Alsmadi O, Melhem M, Hebbar P, Thareja G, John SE, Alkayal F, Behbehani K, Thanaraj TA. Leptin in asscoation with common variants of MC3R mediates hypertension. Am J Hypertens 2014; 27:973-981.

2. Fruhbeck G. Pivotal role of nitric oxide in the control of blood pressure after leptin administration. Diabetes 1999; 48:903-908.

3. Ramchandra R, Barrett CJ, Malpas SC. Nitric oxide and sympathetic nerve activity in the control of blood pressure. Clin Exp Pharmacol Physiol 2005; 32: 440-446.

4. Channanath A, Farran B, Behbehani K, Thanaraj TA. Impact of hypertension on the association of BMI with risk and age at onset of type 2 diabetes mellitus: age- and gender-mediated modifications. PLoS ONE 2014; 9:e95308.

5. Channanath A, Farran B, Behbehani K, Thanaraj TA. State of diabetes, hypertension, and comorbidity in Kuwait. Diabetes Care 2013; 36:e75. 

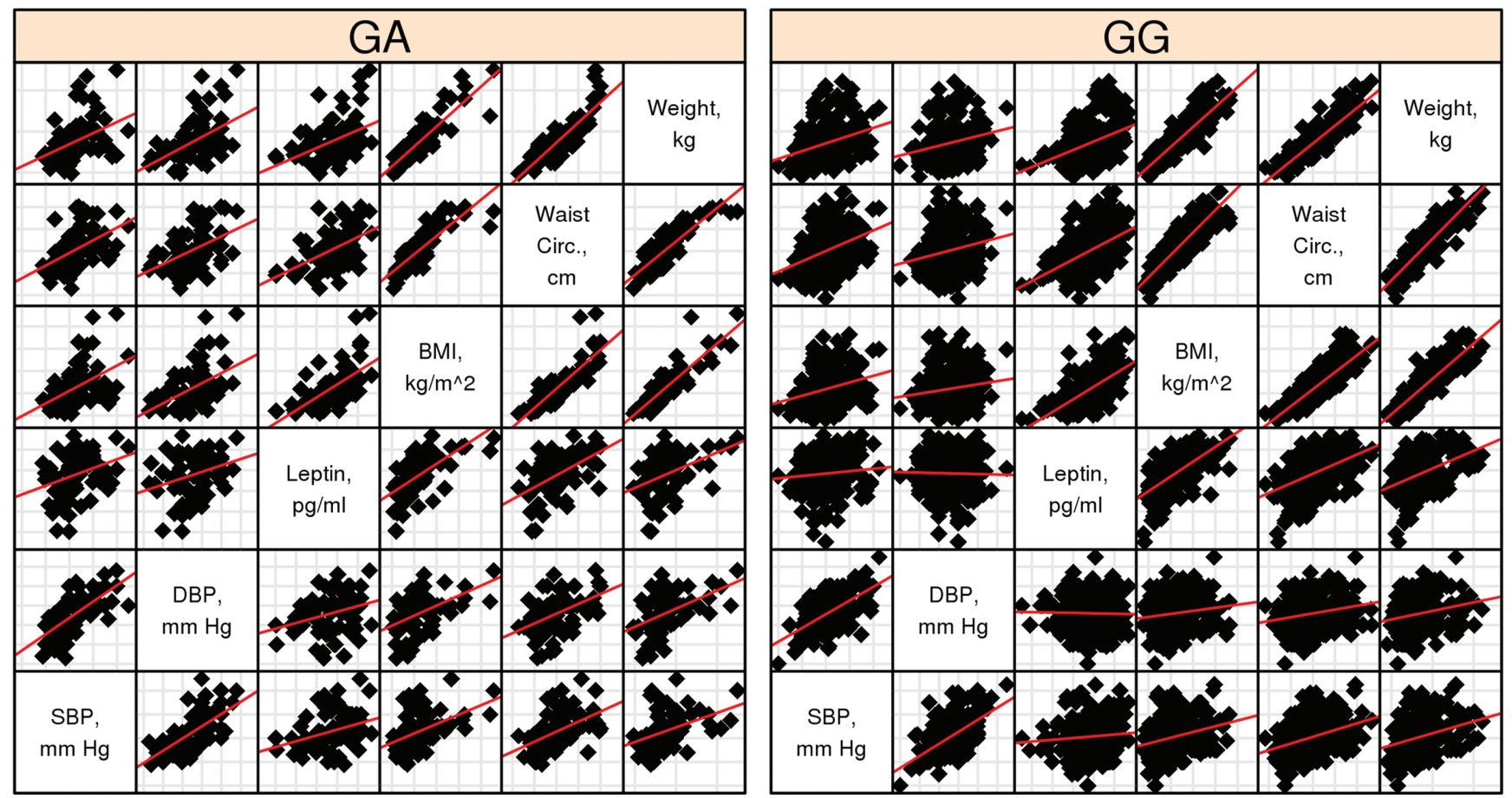

Figure 1. Scatter plot matrix illustrates positive correlation between leptin and obesity traits (BMI, waist circumference, and weight) irrespective of the GG or the variant GA genotypes at rs3827103 on MC3R in the entire study population sample set. However, positive correlation between leptin and SBP ( 3 rd cell from left to right in the bottommost row) occurs only in individuals that harbor the variant (GA). The $R^{2}$, correlation coefficient for SBP vs. leptin in the case of GA is 0.35 and in the case of GG is 0.09 . For further data on other genotypes and co-occurrence of genotypes at rs3746619-rs3827103, see Tables 3 and 4 and Figures 2 and 3 in ref. ${ }^{1}$

Abbreviations: BMI, body mass index; DBP, diastolic blood pressure; SBP, systolic blood pressure. 